\title{
Transient impedance changes in venous endothelial monolayers as a biological radiation dosimetry response
}

\author{
Erik F. Young ${ }^{1,2}$ \\ 1. Center for Radiological Research, Columbia University, New York, United States \\ 2. E-mail any correspondence to: efy1@columbia.edu
}

\begin{abstract}
In March of 2011, a magnitude 9.0 earthquake and subsequent 14 meter high tsunami caused major damage to the Fukushima Daiichi nuclear power plant in Japan. The release of radiation, along with other uncontrolled releases elsewhere, revealed the necessity of a portable high throughput minimally invasive biological dosimetry modality. Immediate and early radiation effects on vasculature could be used as a dosimetry modality. To test whether non-coronary vasculature exhibited transient perturbation in barrier function, video microscopy studies and electric cell-substrate impedance sensing (ECIS) technology were used to probe very subtle changes in primary human vascular endothelium. In our studies, human umbilical vein endothelial cell (HUVEC) monolayers exhibited a transient, significant decrease $(p=0.017)$ in monolayer resistance three hours after irradiation with 5.0 Gy of $\gamma$ rays. Radiation induced perturbations in HUVEC monolayer permeability are similar in magnitude and kinetics to those observed in coronary arterial endothelium. Therefore, at least two types of endothelia respond to radiation on ECIS arrays with an early transient disruption in permeability. This finding supports the use of early passage HUVECs for use in bioelectric dosimetry studies of vasculature and suggests that permeability changes in superficial vessels and sequellae could potentially serve as biological dosimetry tools.
\end{abstract}

Keywords: Bioimpedance, endothelium, ECIS, radiation, dosimetry, HUVEC.

\section{Introduction}

Endothelial cells lining the inner face of blood vessels in the vasculature have a necessary and dynamic barrier function, keeping tissue beds separate from the circulating blood. The failure of the endothelial barrier function in response to radiation allows for factors circulating in the blood to pass detrimentally to the tissue bed. In the context of radiation therapy, this can cause unwanted pathology [1, 2]. A similar modified wound healing response also appears in more superficial tissues and the skin [3, 4]. Common to both processes is the failure of the barrier function of endothelial cells reported by the leakage of tracers from the circulating plasma into the irradiated tissue bed $[5,6]$. One important aspect of some older studies is the use of relatively low energy x-ray sources which permit deposition of energy into more superficial tissues where visible effects in the skin are dependent on the dose employed and the area and proximity of non-irradiated skin [7].
Given its visibility and accessibility, human skin has been extensively studied as a marker of radiation response using colorimetry which non-invasively reports the redness and pigmentation at defined wavelengths of light studied using reflectometry [8-12]. Studies pursuing the overall mechanism of radiation on skin have been carried out in the skin of pigs, a model system frequently used in such comparative studies [13]. These studies reveal that lower doses generate a reddening commensurate with vascular permeability changes. Higher doses that cause greater cell death seem to generate more fulminant inflammation commensurate with cell replacement biology [14-16]. Impedance based strategies are competent to study inflammatory processes of the skin $[17,18]$. An advantage of impedance based strategies is the ability to report not only blood content that is associated with visible redness, but also subtle changes in structural architecture and water content in the interrogated tissue as well. Indeed, radiation induced fibrosis can be quantified by impedance measurements at later time points after exposure $[19,20]$. Current biological dosimetry techniques rely on quantitative assessment of chromosome breaks and rearrangements or the formation of micronuclei in cells stimulated to divide [21-24]. Technologies under development include gene expression and metabolomic approaches which require analysis of body fluids, namely blood or urine [25-27]. Impedance based measurements of skin, as a simple, rapid, and accessible triage modality, could be of great benefit in a radiation emergency response. It is known that endothelial monolayers prepared from the human coronary endothelium respond to ionizing radiation by changing shape and permeability with relatively early kinetics [28]. Other investigators have observed that small sections of vasculature obtained in biopsy can report systemic pathologies of the donor with good fidelity [29]. If the relatively early vascular permeability change seen in coronary monolayers also occurs in cells sourced from other vasculature, such a finding would support the use of bioelectric assessment of vascular changes or the subsequent, structural and water-content changes arising later on in the tissue as a biodosimetry modality.

\footnotetext{
Supplementary files:

https://github.com/joeb-files/2012_Young
} 


\section{Materials and methods}

Cells. Primary HUVECs were a gift from Drs. Joseph Dufraine and Jan Kitajewski of Columbia University. Cells of $\leq 11$ passages were used.

Culture. The HUVECs were seeded at $75 \times 10^{3}$ cells $/ \mathrm{cm}^{2}$ and allowed to mature into confluent monolayers over an 18-20 h period before irradiation and subsequent surveillance. All surfaces were coated with type I collagen (BD, Bedford, MA) and blocked with bovine serum albumin (Sigma, St. Louis, MO) prior to seeding. Cells were maintained in a modified EBM-2 media (Lonza, Rockville, ME) described in earlier work [28].

Irradiation. Cells were insulated from thermal shock and transported to a nearby Gammacell 40 apparatus (Atomic Energy, Ontario, Canada) permitting irradiation with a ${ }^{137} \mathrm{Cs}$ source which delivered 5.0 Gy of $\gamma$ rays at 0.79 $\mathrm{Gy} / \mathrm{min}$.

ECIS recordings. Type " $8 \mathrm{~W} 10 \mathrm{E}+$ " ECIS arrays, connected to an ECIS-Z $\theta$ device (Applied Biophysics, Troy, NY), were used to make multifreqency impedance measurements in two-fold intervals between $62.6 \mathrm{~Hz}$ and $64 \mathrm{kHz}$ at 5 minute intervals permitting assessment of contact, spreading and sealing of the monolayer prior to irradiation, and its response thereafter. For clarity and simplicity, only the ohmic, real resistance at $4 \mathrm{kHz}$ is reported. Furthermore, values collected at $4 \mathrm{kHz}$ are used by several groups studying endothelial biology as a measure of permeability and sealing [30, 31].

Imaging. Time-lapse video microscopy was done using a C4040Z camera mounted to a CK2 microscope (Olympus) via a $20 \mathrm{X}$ phase contrast objective. Recordings were made in a tissue culture incubator at $37^{\circ} \mathrm{C}$ in a humidified $5 \%$ $\mathrm{CO}_{2}$ atmosphere.

\section{Results}

Radiation effects on HUVECs were assessed using electric cell substrate impedance sensing (ECIS) technology. Using this sensitive, continuous bioelectric measurement approach, the laboratory previously described a six-fold decrease in monolayer resistance after wounding and irradiation, observing a statistically significant decrease in resistance after radiation alone [28]. The ECIS instrument passes multiple frequencies of alternating current through gold pads on a planar array affording extremely sensitive, continuous, non-invasive recordings of monolayer resistance $[32,33]$.

\section{Monolayer Changes}

HUVECs were seeded on collagen coated ECIS arrays at values near confluence at the beginning of the experiment. The individual endothelial cells required time to mature into a sealed monolayer with competent barrier function. ECIS recordings of HUVEC monolayers showed cells making contact with the substrate and spreading over an initial 4-6 $\mathrm{h}$ period followed by a slower increase in transmonolayer resistance associated with establishment of lateral contacts and monolayer sealing (Fig 1A). After reaching peak resistance after $14 \mathrm{~h}$ of culture, resistance began to gradually decline. Following irradiation, two phenomena are observed. There was a small increase in resistance reaching a maximum at $1 \mathrm{~h}$ after irradiation and a larger, significant decrease $(n=3 ; p=0.017)$ in monolayer resistance with a maximum occurring roughly $3 \mathrm{~h}$ after irradiation (Fig. 1B). In a separate, independent iteration of the experiment, this maximum shifted later by $0.5 \mathrm{~h}$. Different current paths beneath and between cells, dependent on frequency, inform a modeling algorithm which permits discernment of electrical contributions arising from lateral contacts and cell-substrate contacts (34, 35). There was no statistically significant perturbation in the substrate-interaction parameter, $\alpha$, when models were applied to the monolayers (Fig. S1). The majority of the observed change in resistance is commensurate with the $\mathrm{Rb}$ modeling parameter that reports the resistance arising from lateral contacts between cells (Fig. 1C). Given the suggestion of a decrease in resistance, a single tailed $t$-test was used and found the depression in the $\mathrm{Rb}$ parameter at 3 $\mathrm{h}$ after irradiation to be statistically significant $(n=3 ; p=$ 0.038).
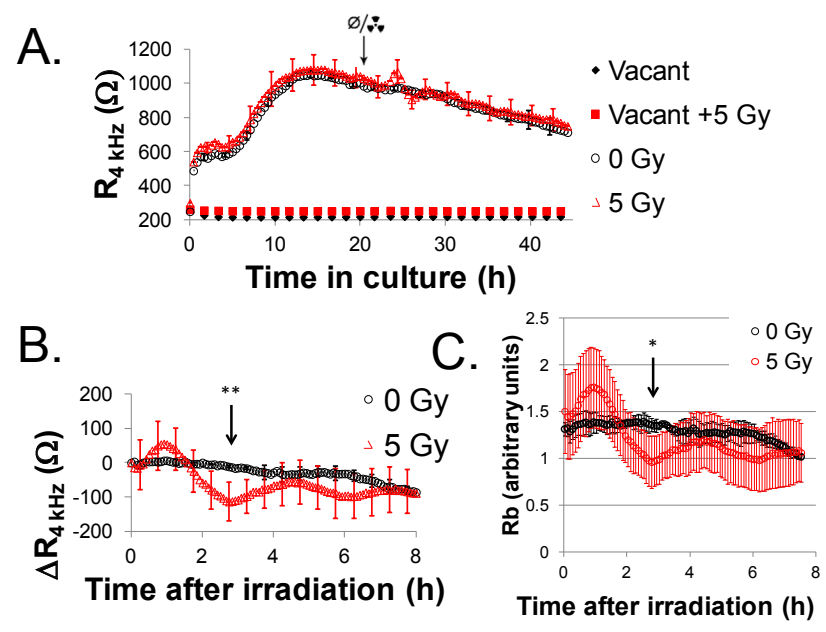

Fig.1: Radiation induced changes in venous endothelial monolayer permeability via ECIS. Primary human umbilical cord vein endothelial cells (HUVECs) were seeded on collagen coated $8 \mathrm{~W} 10 \mathrm{E}+$ arrays and allowed to mature into competent monolayers prior to irradiation. Transmonolayer resistance for the entire course of culture is shown in $A$. The trace shows contact, spreading, sealing and decline of the monolayer's cells over time. Changes in resistance after irradiation shown in $B$ reveal a significant decrease $(* *, p=0.017)$ in monolayer resistance $3 \mathrm{~h}$ after irradiation. The dominant contributor to this resistance is the modeled $\mathrm{Rb}$ parameter $(C)$ that reported a statistically significant $(*, p=0.038)$ change in resistance arising from lateral cell contacts. The mean and standard deviation of three monolayers is plotted. Radiation induces an early, transient decrease in endothelial monolayer permeability.

To verify that electrical changes observed in HUVEC monolayers were associated with changes in permeability and not overt killing or removal of the cells, a time-lapse 
course of video microscopy was performed on irradiated and sham-irradiated controls. Cells were seeded on collagen-coated cultureware at area-corrected densities equivalent to ECIS studies and allowed to mature into competent monolayers before being irradiated. In response to radiation, cells did not appear to retract from neighboring cells in the monolayer. No overt lifting-off or gross changes were observed in the monolayer three hours post irradiation. Later in culture, dead cells were seen to be extruded from the monolayers (Suppl. Movies S2,S3, Fig. 2).

To conclude, HUVEC monolayers, widely used as a model system by many researches, are similar to coronary arterial monolayers in the magnitude and kinetics of radiation induced perturbations to monolayer permeability. Both endothelial monolayer types exhibit a transient 7-8 \% decrease in resistance.

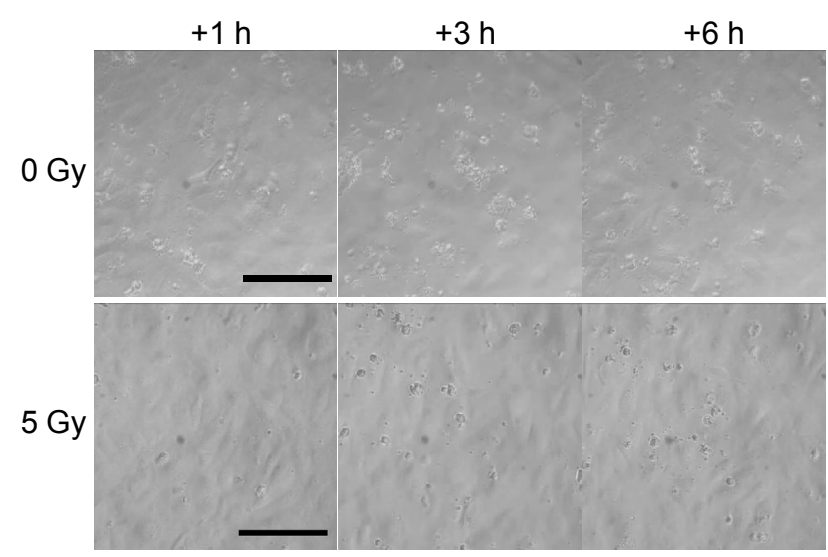

Fig.2: Time-lapse video microscopy of irradiated primary HUVECs. Cells were seeded on collagen-coated substrate and allowed to mature into confluent monolayers before irradiation with 5.0 Gy of $\gamma$ rays. Frames from the video timecourse (Supplementary Movies S2,S3) were captured after irradiation at the intervals shown. Endothelial monolayers are dynamic with dividing and motile cells and are not overtly compromised after radiation. Scale bars; $200 \mu$.

\section{Discussion}

A significant element of any radiation emergency response includes the handling of a population of people who have not received a significant dose of radiation. There is a requirement for a high throughput dosimetry modality to facilitate triage of such a population. The sensitivity of such a modality need not be able to fit existing triage protocols perfectly. These protocols involve grouping of an irradiated population into doses of $<2 \mathrm{~Gy}, 2-6 \mathrm{~Gy}, 6-10 \mathrm{~Gy}$, and $>10$ Gy. A tool to rapidly exclude those exposed to $6 \mathrm{~Gy}$, for example, with a bioelectric measurement could save great deal of time and resources that are otherwise required in a radiation emergency response.

\section{Common versus site-specific responses in vasculature}

Prior work in radiation biology has shown that vascular barriers can be perturbed by irradiation and that this perturbation can be assessed by impedance methods $[6,7$, 28]. The significant and unique finding of this paper, using an impedance method, is that a primary human venous endothelium also responds to gamma irradiation with 5.0 Gy with comparable kinetics to those observed in coronary vascular endothelium. Imaging studies revealed no major changes to the cell monolayer which contrasted with findings in other endothelia which retract more robustly [36, 37].

While a number of endothelial functions are conserved throughout the systemic vascular network, other functions differ depending on location. The endothelium throughout the body varies widely, from delicate sinusoids in the marrow to large capacitance veins in the legs, which, in turn, have a different oxygen and hemodynamic shear exposure. Oxidative species are known to be generated in endothelial cells in response to flow [38]. Oxygen synergizes with ionizing radiation to form damaging reactive oxygen species that damage the cell and so locally elevated oxygen concentration together with greater localized flow conditions could result in a larger effective radiation dose.

Endothelial cells sourced from various sites in the body respond differently to radiation. The most robust response is seen in pulmonary endothelial cells which exhibit significant depolarization of the cytoskeleton and a resultant increase in the wet weight of the lung [36, 37]. Such dramatic responses are not seen in other endothelia [39]. Common endothelial functions do exist and, as seen in the case of rolling of leukocytes across immunologically activated endothelial barriers, these can be reproduced ex vivo [40]. Furthermore the induced relaxation of endothelintreated microvessels seems to be able to report pathologies of the vascular system as a whole [29].

Since oxygen content and hydrodynamic flow conditions differ among venous and arterial vessels, it is important to learn whether primary human umbilical venous endothelial cell (HUVEC) monolayers responded in a different way as compared to an arterial endothelium that would otherwise function at a site of greater oxygen demand. In at least two monolayer systems, sourced from different body sites, impedance changes in response to radiation are very similar and hint at a more widely applicable physiological response that could be utilized by radiation dosimetry.

\section{Dermal vasculature as a biological dosimeter}

The changes in vascular permeability in response to radiation demonstrated here can support two possible dosimetry strategies. The first strategy involves continuous, painless, noninvasive bioelectric surveillance of an individual's physiology. An individual who is under such continuous monitoring could have sensors that report a dose of radiation by sensing vascular permeability changes. The wide use of portable electronic devices together with advances in miniaturization of biomedical sensing 
technologies could make such a strategy feasible in the future. A more feasible strategy at present is to assess the results of a transient change in vascular permeability with a bioimpedance approach. In particular, changes in blood content, fluid content or other tissue architecture changes that are not limited to a seemingly early and transient signal. Such assessment is not without difficulty. It can be argued that the heterogeneity and quality of skin in the human population is significant - varying with age and body site $[41,42]$. Strategies to overcome this heterogeneity do exist. Indeed, normalization against high frequency impedance values has been used in previous studies [17].

This work is limited to a single radiation dose of $5.0 \mathrm{~Gy}$ in a demonstration of the response in a second type of endothelium. Further validation of this response as a dosimetry modality must necessarily include a greater range of doses to establish the threshold of distinction and the threshold of detection. Prior work demonstrates that the leaking of blood from superficial vessels in rodents occurs more rapidly as the radiation dose increases [43]. As radiation doses increase, it is unclear whether the vascular barrier will become opened to a greater extent (amplitude of response) or will remain open longer (duration of response). In future dose-response studies, this question will assuredly be answered. This work supports the idea that sourcing endothelial cells from more amenable sites in the body other than the coronary circulation is possible to allow for assessment of this response in future experiments. The exvivo platform could also provide a means to further probing the molecular mechanisms behind radiation-induced change in the function and shape of endothelial cells.

\section{References}

1. Cottin Y, Kollum M, Kolodgie FD, Chan RC, Kim HS, Vodovotz Y, et al. Intravascular radiation accelerates atherosclerotic lesion formation of hypercholesteremic rabbits. Cardiovasc Radiat Med 2001;2(4):231-40. http://dx.doi.org/10.1016/S1522-1865(02)00129-4

2. Huff H, Sanders EM. Coronary-artery occlusion after radiation. N Engl J Med 1972;286(14):780. http://dx.doi.org/10.1056/NEJM197204062861414

3. Dimitrievich GS, Hausladen SL, Kuchnir FT, Griem ML. Radiation damage and subendothelial repair to rabbit ear chamber microvasculature. An in vivo and histologic study. Radiat Res 1977;69(2):276-92.

http://dx.doi.org/10.2307/3574436

4. Griem ML, Robotewskyj A, Nagel RH. Potential vascular damage from radiation in the space environment. Adv Space Res 1994;14(10):555-63. http://dx.doi.org/10.1016/0273-1177(94)90510-X

5. Jolles B, Harrison RG. Radiation skin reaction and depletion and restoration of body immune response. Nature 1963;198:1216-7. http://dx.doi.org/10.1038/1981216a0

6. Song CW, Anderson RS, Tabachnick J. Early effects of beta irradiation on dermal vascular permeability to plasma proteins. Radiat Res 1966;27(4):604-15. http://dx.doi.org/10.2307/3571844
7. Jolles B. The reciprocal vicinity effect of irradiated tissues on a diffusible substance in irradiated tissues. Br J Radiol 1950;23(265):18-24, illust. http://dx.doi.org/10.1259/0007-1285-23-265-18

8. Gibson IM. Measurement of skin colour in vivo. J. Soc. Cosmet.Chem. 1971;22(11):725-740.

9. Turesson I, Notter G. Dose-response and dose-latency relationships for human skin after various fractionation schedules. Br J Cancer Suppl 1986;7:67-72.

10. Turesson I, Notter G. The influence of fraction size in radiotherapy on the late normal tissue reaction--I: Comparison of the effects of daily and once-a-week fractionation on human skin. Int J Radiat Oncol Biol Phys 1984;10(5):593-8. http://dx.doi.org/10.1016/0360-3016(84)90289-X

11. Turesson I, Notter G. The influence of fraction size in radiotherapy on the late normal tissue reaction--II: Comparison of the effects of daily and twice-a-week fractionation on human skin. Int J Radiat Oncol Biol Phys 1984;10(5):599-606. http://dx.doi.org/10.1016/0360-3016(84)90290-6

12. Turesson I, Notter G. The influence of the overall treatment time in radiotherapy on the acute reaction: comparison of the effects of daily and twice-a-week fractionation on human skin. Int J Radiat Oncol Biol Phys 1984;10(5):607-18. http://dx.doi.org/10.1016/0360-3016(84)90291-8

13. Montagna W, Yun JS. The Skin Of The Domestic Pig. J Invest Dermatol 1964;42:11-21.

14. Fajardo LF. Pathology of Radiation Injury. New York: Masson; 1982.

15. Peel DM, Hopewell JW, Wells J, Charles MW. Late nonstochastic changes in pig skin after beta irradiation. Radiat Res 1985;101(3):491-6. http://dx.doi.org/10.2307/3576508

16. Peel DM, Hopewell JW, Wells J, Charles MW. Nonstochastic effects of different energy beta emitters on pig skin. Radiat Res 1984;99(2):372-82. http://dx.doi.org/10.2307/3576380

17. Ollmar S, Emtestam L. Electrical impedance applied to noninvasive detection of irritation in skin. Contact Dermatitis 1992;27(1):37-42. http://dx.doi.org/10.1111/j.1600-0536.1992.tb05195.x

18. Ollmar S, Nyren M, Nicander I, Emtestam L. Electrical impedance compared with other non-invasive bioengineering techniques and visual scoring for detection of irritation in human skin. Br J Dermatol 1994;130(1):29-36. http://dx.doi.org/10.1111/j.1365-2133.1994.tb06878.x

19. Lahtinen T, Nuutinen J, Alanen E, Turunen M, Nuortio L, Usenius T, et al. Quantitative assessment of protein content in irradiated human skin. Int J Radiat Oncol Biol Phys 1999;43(3):635-8. http://dx.doi.org/10.1016/S0360-3016(98)00439-8

20. Nuutinen J, Lahtinen T, Turunen M, Alanen E, Tenhunen M, Usenius $\mathrm{T}$, et al. A dielectric method for measuring early and late reactions in irradiated human skin. Radiother Oncol 1998;47(3):249-54. http://dx.doi.org/10.1016/S0167-8140(97)00234-X 
21. Flegal FN, Devantier Y, Marro L, Wilkins RC. Validation of QuickScan dicentric chromosome analysis for high throughput radiation biological dosimetry. Health Phys 2012;102(2):143-53. http://dx.doi.org/10.1097/HP.0b013e3182307758

22. Garty G, Chen Y, Turner HC, Zhang J, Lyulko OV, Bertucci A, et al. The RABiT: a rapid automated biodosimetry tool for radiological triage. II. Technological developments. Int J Radiat Biol 2011;87(8):776-90. http://dx.doi.org/10.3109/09553002.2011.573612

23. Turner HC, Brenner DJ, Chen Y, Bertucci A, Zhang J, Wang $\mathrm{H}$, et al. Adapting the gamma-H2AX assay for automated processing in human lymphocytes. 1. Technological aspects. Radiat Res 2011;175(3):282-90. http://dx.doi.org/10.1667/RR2125.1

24. Wilkins RC, Romm H, Oestreicher U, Marro L, Yoshida MA, Suto Y, et al. Biological Dosimetry by the Triage Dicentric Chromosome Assay - Further validation of International Networking. Radiat Meas 2011;46(9):923-928. http://dx.doi.org/10.1016/j.radmeas.2011.03.012

25. Coy SL, Cheema AK, Tyburski JB, Laiakis EC, Collins SP, Fornace A, Jr. Radiation metabolomics and its potential in biodosimetry. Int J Radiat Biol 2012;87(8):802-23. http://dx.doi.org/10.3109/09553002.2011.556177

26. Paul S, Amundson SA. Development of gene expression signatures for practical radiation biodosimetry. Int J Radiat Oncol Biol Phys 2008;71(4):1236-1244.

http://dx.doi.org/10.1016/j.ijrobp.2008.03.043

27. Templin T, Paul S, Amundson SA, Young EF, Barker CA, Wolden SL, et al. Radiation-induced micro-RNA expression changes in peripheral blood cells of radiotherapy patients. Int J Radiat Oncol Biol Phys 2012;80(2):549-57. http://dx.doi.org/10.1016/j.ijrobp.2010.12.061

28. Young EF, Smilenov LB. Impedance-based surveillance of transient permeability changes in coronary endothelial monolayers after exposure to ionizing radiation. Radiat Res 2011;176(4):415-24. http://dx.doi.org/10.1667/RR2665.1

29. Phillips SA, Hatoum OA, Gutterman DD. The mechanism of flow-induced dilation in human adipose arterioles involves hydrogen peroxide during CAD. Am J Physiol Heart Circ Physiol 2007;292(1):H93-100. http://dx.doi.org/10.1152/ajpheart.00819.2006

30. Tiruppathi C, Malik AB, Del Vecchio PJ, Keese CR, Giaever I. Electrical method for detection of endothelial cell shape change in real time: assessment of endothelial barrier function. Proc Natl Acad Sci U S A 1992;89(17):7919-23. http://dx.doi.org/10.1073/pnas.89.17.7919

31. van Wetering S, van den Berk N, van Buul JD, Mul FP, Lommerse I, Mous R, et al. VCAM-1-mediated Rac signaling controls endothelial cell-cell contacts and leukocyte transmigration. Am J Physiol Cell Physiol 2003;285(2):C343-52.
32. Giaever I, Keese CR. Monitoring fibroblast behavior in tissue culture with an applied electric field. Proc Natl Acad Sci U S A 1984;81(12):3761-4. http://dx.doi.org/10.1073/pnas.81.12.3761

33. Giaever I, Keese CR. A morphological biosensor for mammalian cells. Nature 1993;366(6455):591-2. http://dx.doi.org/10.1038/366591a0

34. Moy AB, Winter M, Kamath A, Blackwell K, Reyes G, Giaever I, et al. Histamine alters endothelial barrier function at cell-cell and cell-matrix sites. Am J Physiol Lung Cell Mol Physiol 2000;278(5):L888-98.

35. Giaever I, Keese CR. Micromotion of mammalian cells measured electrically. Proc Natl Acad Sci U S A 1991;88(17):7896-900. http://dx.doi.org/10.1073/pnas.88.17.7896

36. Kantak SS, Diglio CA, Onoda JM. Low dose radiationinduced endothelial cell retraction. Int J Radiat Biol 1993;64(3):319-28. http://dx.doi.org/10.1080/09553009314551471

37. Onoda JM, Kantak SS, Diglio CA. Radiation induced endothelial cell retraction in vitro: correlation with acute pulmonary edema. Pathol Oncol Res 1999;5(1):49-55. http://dx.doi.org/10.1053/paor.1999.0049

38. Liu Y, Zhao H, Li H, Kalyanaraman B, Nicolosi AC, Gutterman DD. Mitochondrial sources of $\mathrm{H} 2 \mathrm{O} 2$ generation play a key role in flow-mediated dilation in human coronary resistance arteries. Circ Res 2003;93(6):573-80. http://dx.doi.org/10.1161/01.RES.0000091261.19387.AE

39. Gabrys D, Greco O, Patel G, Prise KM, Tozer GM, Kanthou C. Radiation effects on the cytoskeleton of endothelial cells and endothelial monolayer permeability. Int J Radiat Oncol Biol Phys 2007;69(5):1553-62. http://dx.doi.org/10.1016/j.ijrobp.2007.08.039

40. Vereycken-Holler V, Aigueperse J, Gaugler MH. Radiation effects on circulating and endothelial cell interactions studied by quantitative real-time videomicroscopy. Int J Radiat Biol 2002;78(10):923-30. http://dx.doi.org/10.1080/09553000210152980

41. Panescu P, Cohen KP, Webster JG, Stratbucker RA. The mosaic electrical characteristics of the skin. Biomedical Engineering, IEEE Transactions on 1993;40(5):434.

42. Pearce RH, Grimmer BJ. Age and the chemical constitution of normal human dermis. J Invest Dermatol 1972;58(6):34761. http://dx.doi.org/10.1111/1523-1747.ep12540531

43. Jolles B. X-ray Skin Reactions and the Protective Role of Normal Tissues. Br J Radiol 1941;14(159):110-112. http://dx.doi.org/10.1259/0007-1285-14-159-110 